ARTIGO - ARTICLE - ARTÍCULO

\title{
Violência contra crianças e adolescentes na Bahia: caracterização dos casos, 2009-2016
}

Violence against children and adolescents in Bahia: characterization of cases, 2009-2016

Violencia contra niños, niñas y adolescentes en Bahía: caracterización de casos, 2009-2016

\author{
Izabel Cristina Neves Ramos (D), Thaís Regis Aranha Rossi (D)
}

Universidade do Estado da Bahia (UNEB), Salvador, Bahia, Brasil

\section{RESUMO}

O estudo teve como objetivo caracterizar, através de uma série histórica, o quadro da violência contra crianças e adolescentes no estado da Bahia por meio da análise das notificações realizadas pelos serviços de saúde. Foram utilizados dados do Sistema de Vigilância de Violência e Acidentes (VIVA Contínuo), durante os anos de 2009 a 2016, totalizando 14.115 casos reportados. Os dados mostraram um aumento de 212,30\% nas notificações, entre 2009 e 2016. Quanto às vítimas, a faixa etária de 10 a 19 anos prevaleceu em todos os anos analisados, assim como a presença de vítimas autodeclaradas negras. A tipologia mais frequente foi violência física. Para análise estatística, foi utilizado software Minitab18. Os achados desde estudo são preocupantes e demonstram a magnitude da violência contra crianças e adolescentes, bem como destacam suas especificidades e a importância do combate a esse problema de saúde.

Palavras-chave: Violência; Serviços de saúde; Crianças; Adolescente.

\begin{tabular}{|c|c|}
\hline \multicolumn{2}{|c|}{ Histórico do Artigo } \\
\hline ecebido & 01 Outubro 2020 \\
\hline provado & 020 \\
\hline \multicolumn{2}{|c|}{ Correspondência } \\
\hline \multicolumn{2}{|c|}{ Thaís Regis Aranha Rossi } \\
\hline \multicolumn{2}{|c|}{ Universidade do Estado da Bahia-UNEB } \\
\hline \multicolumn{2}{|c|}{ Departamento de Ciências da Vida-DCV } \\
\hline \multicolumn{2}{|c|}{ Rua Silveira Martins, 2555, Cabula } \\
\hline \multicolumn{2}{|c|}{ CEP 41.150-000 - Salvador, Bahia. } \\
\hline \multicolumn{2}{|c|}{ E-mail: thais.aranha@gmail.com } \\
\hline \multicolumn{2}{|l|}{ Como citar } \\
\hline \multicolumn{2}{|c|}{$\begin{array}{l}\text { Ramos ICN, Rossi TRA. Violência contra } \\
\text { crianças e adolescentes na Bahia: carac- } \\
\text { terização dos casos de } 2009 \text { a } 2016 . \\
\text { Rev. Saúde Col. UEFS 11(1): e5899. }\end{array}$} \\
\hline
\end{tabular}




\section{INTRODUÇÃO}

A violência é um fenômeno sócio-histórico que acompanha a trajetória da humanidade ${ }^{1}$, atinge populações de todas as culturas e apresenta diferenciais entre os gêneros, classes e etnias. Segundo definição da Organização Mundial da Saúde (OMS), a violência é o uso intencional da força física ou do poder real, ou em ameaça contra si próprio ou contra outra pessoa, grupo ou comunidade, resultando, ou que tenha a possibilidade de resultar, em lesão, morte ou dano psicológico, deficiência de desenvolvimento ou privação ${ }^{2}$. Esse agravo representa uma das principais causas de morte no Brasil e mesmo quando não deixa marcas físicas, produz efeitos que acompanham o indivíduo durante toda a sua vida $^{3,4}$, podendo ainda ser reproduzida na forma como as crianças lidam com conflitos futuros 5 .

A violência causa ainda impacto na capacidade produtiva da sociedade ${ }^{6}$ e, segundo dados do Atlas da Violência produzido pelo Instituto de Pesquisa Econômica Aplicada (IPEA) em conjunto com o Fórum Brasileiro de Segurança Pública (FBSP), entre 2008 e 2017, a violência intencional causou a morte de 553.000 pessoas no Brasil, atingindo principalmente homens jovens (de 15 a 19 anos), para quem o homicídio foi responsável por 56,5\% dos óbitos, neste grupo, em 2017. Segundo este mesmo documento, a Bahia encontra-se entre as Unidades Federativas (UF) que possuem as maiores taxas de homicídios, com 46,9 óbitos a cada 100 mil habitantes. A Bahia também figura entre os três estados com maior porcentagem $(10,8 \%)$ de Mortes Violentas por Causa Indeterminada (MVCI), ficando atrás apenas de Minas Gerais $(11,0 \%)^{7}$.

As consequências da violência podem impactar também nos serviços de saúde, quando demandam atendimentos ambulatoriais e/ou internações hospitalares ${ }^{1,4}$. Por conta destas repercussões na saúde individual e coletiva, a violência hoje é considerada um problema de saúde pública. Porém, ainda carecem estudos acerca da violência infantil em âmbitos nacional e estadual ${ }^{1}$. Com isso, faz-se importante a realização de pesquisas com o objetivo de entender como a violência infantil se comporta ao longo do tempo para que estratégias de prevenção e enfrentamento possam ser elaboradas e para que as estratégias já em curso possam ser acompanhadas e avaliadas quanto à sua efetividade ${ }^{6}$.

Antes da implementação do Sistema de Vigilância de Violência e Acidentes (VIVA), as informações sobre violência eram obtidas principalmente através do Sistema de Informações sobre Mortalidade (SIM) e do Sistema de Informações Hospitalares (SIH), porém era necessário ampliar as informações, principalmente sobre os atendimentos por conta de violências e acidentes realizados nas unidades de emergência. Para isso, em 2006, o Ministério da Saúde implementou o Sistema VIVA cujo objetivo foi utilizar as informações coletadas para subsidiar políticas em saúde pública voltadas para violências e acidentes, além de auxiliar na melhoria do serviço prestado às vítimas desses agravos ${ }^{6}$.
O Sistema VIVA é dividido em dois componentes: o Contínuo e o Inquérito/Sentinela. O VIVA Contínuo (Vigilância de violência doméstica, sexual, e/ou outras violências interpessoais e autoprovocadas) integra o Sistema de Informação de Agravos de Notificação (SINAN), desde 2009. Já, o VIVA Inquérito/Sentinela (Vigilância de violências e acidentes em emergências hospitalares) é um estudo transversal, realizado a cada dois anos, em um período de 30 dias consecutivos, em serviços de urgência e emergência selecionados. A implementação do sistema VIVA é de responsabilidade das Secretarias Municipais e Estaduais de Saúde, SMS e SES, respectivamente ${ }^{8}$.

Os estudos ${ }^{8,9}$ que vêm sendo realizados apontam um alto índice de subnotificação dos casos de violência infantil com predominância do sexo masculino entre as vítimas e agressores, exceto na violência sexual, onde as vítimas são majoritariamente crianças do sexo feminino ${ }^{4,8,9}$. Apesar de não ser o objetivo deste estudo, alguns estudos ${ }^{4,10}$ mostram associação entre a ocorrência de violência em via pública e as vítimas do sexo masculino uma vez que, em uma sociedade com desigualdade de gênero, os meninos possuem mais liberdade para transitar nas ruas enquanto as meninas estão no ambiente domiciliar ${ }^{4,10,17}$.

Pesquisas apontam também para uma diferença entre raça/cor, onde os negros (pretos e pardos) apresentam-se como as maiores vítimas ${ }^{10,11}$. A literatura aponta ainda uma diferença entre a tipologia da violência e a idade da vítima, sendo a negligência mais evidenciada em crianças menores de 2 anos $^{10}$.

Existem poucos estudos que fazem uma caracterização da violência utilizando o VIVA Contínuo que proporciona uma análise mais abrangente, uma vez que fornece dados mais completos por ser fruto da análise durante um ano, enquanto o inquérito diz respeito apenas à coleta de um mês. Nenhuma série histórica do estado da Bahia, com a amplitude realizada por esta pesquisa foi encontrada na literatura, para as faixas etárias da infância e adolescência.

Este estudo busca caracterizar, através de uma série histórica, o quadro da violência infantil na Bahia, tendo como ponto de partida os casos notificados e disponibilizados através do Sistema VIVA Contínuo, entre os anos de 2009 e 2016. Com essa caracterização, pretende-se contribuir para o conhecimento sobre a temática, trazendo o debate e buscando intensificar o enfrentamento à violência contra a criança e o adolescente, contribuindo para a cultura da paz tanto no cenário nacional quanto no cenário internacional. Ademais, busca-se contribuir com o monitoramento desse sistema de informações e das políticas voltadas a estas intervenções.

\section{METODOLOGIA}

Foi feita uma análise descritiva de todas as notificações de casos de violência psicológica/moral, negligência/abandono, física, tortura, sexual, tráfico de seres humanos e trabalho 
infantil, realizadas em qualquer espaço, inclusive em ambiente escolar, contra crianças e adolescentes de distintas faixas etárias (0 a 19 anos) registradas no SINAN, na Bahia, no período de 2009 a 2016. Como na base de amplo acesso (TABNET DATASUS) estavam disponíveis apenas os anos de 2009 a 2011, foi feito contato direto com o Ministério da Saúde que disponibilizou os bancos de dados de 2009 a 2016.

A ficha de notificação consiste, principalmente, em informações gerais (como a UF e a Unidade de Notificação), informações sobre o paciente, informações sobre a violência e informações sobre o agressor. Os critérios de exclusão foram: casos de violência nos quais as vítimas tinham idade superior a 19 anos e casos em que a lesão foi tida como autoprovocada. Foram analisadas as variáveis classificadas em relação à vítima: sexo (feminino ou masculino), faixa etária (em ciclos de vida, de acordo com a classificação do VIVA), raça/cor (branca, parda, preto, amarela, indígena), zona de residência (urbana, rural ou periurbana), deficiência (sim, não, não se aplica); ao agressor: sexo (masculino, feminino, ambos os sexos), vínculo com a vítima (pai/mãe, padrasto, irmão, amigo/conhecido, desconhecido, outros), suspeita de uso de álcool (sim ou não); quanto à ocorrência: local (residência, escola, via pública, outros), zona (urbana, rural ou periurbana), recorrência, tipo de violência, natureza da agressão, natureza da lesão, parte do corpo atingida, procedimento realizado no caso de violência sexual, consequência causada pela violência, encaminhamento realizado, evolução do caso, conforme registro feito por profissional de saúde que atendeu o caso suspeito ou confirmado de violência.

Houve uma mudança na ficha de notificação em 2014, o que impossibilitou a análise de algumas variáveis nos anos de 2015 e 2016 (estes últimos anos estão também sujeitos a alterações pelo Ministério da Saúde). Quanto aos dados de 2017 a 2020, ainda não haviam sido disponibilizados até a data da submissão do artigo. A análise estatística dos dados foi realizada no software MINITAB 18.

\section{RESULTADOS}

Conforme a Tabela 1, houve um total de 14.115 casos notificados no estado da Bahia, no período de 2009 a 2016. Em 2009, foram 789 casos notificados e em 2016 este número passa a ser 2.464, representando um aumento de $212,30 \%$ nas notificações.

Tabela 1. Caracterização sociodemográfica das vítimas de violência contra crianças e adolescentes, Bahia, 2009-2016

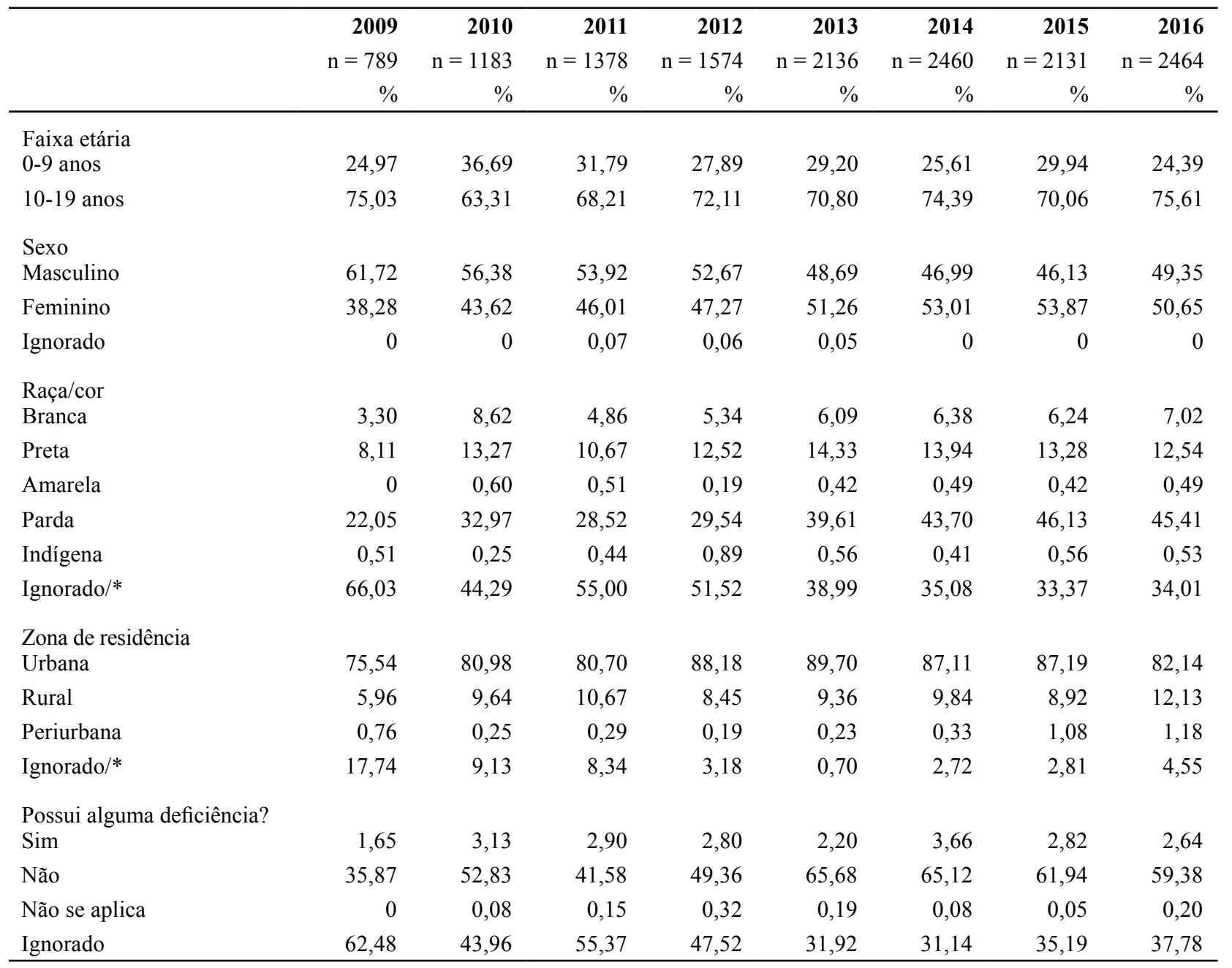


Em todos os anos, a faixa etária de 10-19 anos foi a mais prevalente. Quanto ao sexo das vítimas, observa-se um predomínio das vítimas do sexo masculino, de 2009 a 2012, e predomínio do sexo feminino, entre 2013 e 2016. Negros foram as maiores vítimas de violência em todos os anos e observa-se um aumento no número de vítimas pardas, assim como uma diminuição no número de ignorados: de $66,03 \%$, em 2009, para 34,01\%, em 2016. Quanto à zona de residência, observa-se um aumento que diz respeito às vítimas da zona rural, de 5,96\%, em 2009, para 12,13\%, em 2016.
Crianças e adolescentes com algum tipo de deficiência ou transtorno também foram vítimas das violências, correspondendo a 1,65\% das ocorrências de 2009 e 2,64\%, de 2016.

A maioria das ocorrências foi na zona urbana (Tabela 2), em todos os anos, sendo observada uma queda no número de ignorados: 53,23\% em 2009 e 21,95\% em 2016. No que tange ao local de ocorrência, destaca-se a prevalência das residências das vítimas, com um aumento de 21,42\%, em 2009, para $42,86 \%$, em 2010, e certa estabilização nos anos subsequentes (32,29\%, em 2011; 39,37\%, em 2013; e 32,95, em 2016).

Tabela 2. Caracterização da violência contra crianças e adolescentes segundo local, zona e desfechos das ocorrências, Bahia, 2009-2016

\begin{tabular}{|c|c|c|c|c|c|c|c|c|}
\hline & $\begin{array}{c}2009 \\
\mathrm{n}=789 \\
\%\end{array}$ & $\begin{array}{c}2010 \\
\mathrm{n}=1183 \\
\%\end{array}$ & $\begin{array}{c}2011 \\
\mathrm{n}=1378 \\
\%\end{array}$ & $\begin{array}{c}2012 \\
\mathrm{n}=1574 \\
\%\end{array}$ & $\begin{array}{c}2013 \\
\mathrm{n}=2136 \\
\%\end{array}$ & $\begin{array}{c}2014 \\
\mathrm{n}=2460 \\
\%\end{array}$ & $\begin{array}{c}2015 \\
\mathrm{n}=2131 \\
\%\end{array}$ & $\begin{array}{c}2016 \\
\mathrm{n}=2464 \\
\%\end{array}$ \\
\hline \multicolumn{9}{|l|}{ Zona de ocorrência } \\
\hline Urbana & 40,81 & 61,03 & 42,31 & 55,59 & 62,22 & 63,05 & 73,44 & 71,27 \\
\hline Rural & 4,56 & 8,37 & 9,8 & 7,56 & 7,91 & 9,47 & 9,57 & 11,85 \\
\hline Periurbana & 1,01 & 0,68 & 0,51 & 0,38 & 0,56 & 0,61 & 1,64 & 1,22 \\
\hline Ignorado/* & 53,62 & 29,92 & 47,38 & 36,47 & 29,31 & 26,87 & 15,35 & 15,66 \\
\hline \multicolumn{9}{|l|}{ Local de ocorrência } \\
\hline Residência & 21,42 & 42,86 & 32,29 & 36,15 & 39,37 & 38,25 & 36,04 & 32,95 \\
\hline Escola & 1,01 & 2,03 & 1,31 & 2,16 & 1,54 & 1,83 & 2,77 & 2,11 \\
\hline Via pública & 18,5 & 13,95 & 12,12 & 17,28 & 23,36 & 23,98 & 22,85 & 32,63 \\
\hline Outro & 5,83 & 8,45 & 6,68 & 5,77 & 11,18 & 8,75 & 10,27 & 10,35 \\
\hline Ignorado & 53,24 & 32,71 & 47,6 & 38,64 & 24,55 & 27,19 & 28,07 & 21,96 \\
\hline \multicolumn{9}{|c|}{ Procedimento realizado no caso de violência sexual } \\
\hline Profilaxia DST & 3,17 & 3,8 & 4,14 & 4,51 & 3,79 & 4,51 & 5,11 & 5,64 \\
\hline Profilaxia HIV & 2,92 & 3,3 & 3,56 & 3,88 & 3,37 & 3,74 & 4,6 & 4,83 \\
\hline Profilaxia Hepatite B & 1,65 & 2,37 & 2,61 & 2,16 & 2,25 & 2,76 & 3 & 2,68 \\
\hline Coleta de sangue & 3,55 & 4,23 & 4,5 & 5,72 & 4,07 & 5,37 & 6,99 & 7,02 \\
\hline Coleta de sêmen & 0 & 0,25 & 0,65 & 0,7 & 0,23 & 0,49 & 0,52 & 0,32 \\
\hline Coleta de secreção vaginal & 0,38 & 1,01 & 0,87 & 0,89 & 0,61 & 1,02 & 0,99 & 0,93 \\
\hline Contracepção de emergência & 0,76 & 1,1 & 1,74 & 2,1 & 2,53 & 2,32 & 2,63 & 2,96 \\
\hline Aborto previsto em lei & 0 & 0 & 0,07 & 0,06 & 0,09 & 0,24 & 0,23 & 0,28 \\
\hline \multicolumn{9}{|c|}{ Consequências da ocorrência detectadas no momento da notificação } \\
\hline Gravidez & 0,63 & 1,1 & 0,51 & 0,95 & 1,08 & 0,98 & S.I & S.I \\
\hline DST & 0,89 & 1,61 & 0,87 & 1,14 & 1,22 & 1,26 & S.I & S.I \\
\hline Tentativa de suicídio & 0,13 & 0,34 & 0,29 & 0,19 & 0,19 & 0,37 & S.I & S.I \\
\hline Transtorno mental & 0,13 & 0,68 & 0,36 & 0,25 & 0,56 & 0,28 & S.I & S.I \\
\hline Transtorno comportamental & 3,3 & 4,48 & 4,35 & 3,11 & 3,46 & 2,07 & S.I & S.I \\
\hline Estresse pós-traumático & 6,59 & 4,48 & 5,95 & 6,35 & 7,82 & 4,27 & S.I & S.I \\
\hline Outros & 4,18 & 5,41 & 2,76 & 2,35 & 1,36 & 1,38 & S.I & S.I \\
\hline \multicolumn{9}{|l|}{ Encaminhamento no setor saúde } \\
\hline Encaminhamento ambulatorial & 16,86 & 23,33 & 18,36 & 23,63 & 25,89 & S.I & S.I & S.I \\
\hline Internação hospitalar & 68,57 & 45,56 & 53,92 & 52,16 & 41,99 & S.I & S.I & S.I \\
\hline Não se aplica & 3,55 & 7,95 & 8,49 & 12,58 & 17,42 & S.I & S.I & S.I \\
\hline Ignorado & 11,02 & 23,16 & 19,23 & 11,63 & 14,7 & S.I & S.I & S.I \\
\hline \multicolumn{9}{|l|}{ Evolução do caso } \\
\hline Alta & 66,03 & 68,89 & 65,09 & 68,74 & 71,16 & 61,3 & S.I & S.I \\
\hline Evasão / Fuga & 0,38 & 0,51 & 0,87 & 0,95 & 0,8 & 1,06 & S.I & S.I \\
\hline Óbito por Violência & 0,51 & 3,63 & 3,63 & 2,86 & 1,97 & 1,26 & S.I & S.I \\
\hline Óbito por outras causas & 0,25 & 0,25 & 0,15 & 0,13 & 0,14 & 0,04 & S.I & S.I \\
\hline Ignorado & 32,83 & 26,72 & 30,26 & 27,32 & 25,93 & 36,34 & S.I & S.I \\
\hline
\end{tabular}

Fonte: VIVA/SVS/MS 
A via pública como local da violência merece um destaque, tendo em vista que vem se apresentando de forma crescente com 18,50\% de casos, em 2009; 17,28\%, em 2012; 23,36\%, em 2013, e 32,63\%, no ano de 2016 .

Quanto aos procedimentos realizados nos casos de violência sexual, expostos na Tabela 2, houve um crescimento nas profilaxias realizadas para DST distribuindo-se em 3,17\% dos casos na violência sexual notificada, em 2009, e chegando a $5,64 \%$, em 2016. Esse crescimento também pôde ser observado nas profilaxias para HIV e Hepatite B, tendo a profilaxia para HIV passando de 2,92\%, em 2009, para 4,83\%, em 2016, e a Hepatite B de 1,65\%, em 2009, para 2,68\%, em 2016. A contracepção de emergência e o aborto também cresceram, tendo a contracepção de emergência apresentado a seguinte distribuição: 0,76\%, em 2009, e 2,96\%, em 2016. Quanto ao aborto previsto em lei, não houve notificações do procedimento nos anos de 2009 e 2010. Já em 2011, o aborto previsto em lei correspondeu a $0,07 \%$ dos procedimentos realizados nos casos de violência sexual e, em 2016, este procedimento foi realizado em $0,28 \%$ dos casos de violência sexual.

$\mathrm{Na}$ análise do encaminhamento realizado no setor saúde, presentes na Tabela 2, observa-se um alto percentual de internações hospitalares: $68,57 \%$, no ano de $2009 ; 45,56 \%$, no ano de $2010 ; 53,92 \%$, no ano de $2011 ; 52,16 \%$, no ano de 2012 ; e $41,99 \%$, no ano de 2013 . Os encaminhamentos ambulatoriais tiveram um aumento no percentual de casos, passando de $16,86 \%$, em 2009, para 25,89\%, em 2013. Os anos de 2014, 2015 e 2016 não estavam disponíveis. Quanto à evolução do caso, alta foi o desfecho mais frequente, mantendo uma regularidade durante os anos: $66,03 \%$, em 2009; 68,89\%, em 2010; 65,09\%, em $2011 ; 68,74 \%$, em 2012; 71,16\%, em 2013; e 61,30\%, em 2014. Outro desfecho importante é a evasão/fuga, que apresentou um incremento ao longo dos anos: 0,38\%, em 2009 e 1,06\%, em 2014. Os anos de 2015 e 2016 não estavam disponíveis.

Segundo a tipologia da violência (Tabela 3), houve grande predomínio da violência física em todos os anos, conforme exposto na Tabela 2, com 86,8\%, em 2009, e $76,38 \%$, em 2016. O segundo tipo de violência mais frequente foi a psicológica/moral, com $12,93 \%$ dos casos notificados em 2009; 24,71\%, em 2012; e 18,55\%, em 2016.

Foram também notificados casos de tráfico de seres humanos, sendo 2011 o ano com maior número de casos notificados, representando $0,29 \%$. Houve grande número de casos de violência sexual: $11,66 \%$, no ano de $2009 ; 18,80 \%$ das ocorrências do ano de 2011; 27,43\%, em 2013; 28,25\%, em 2015 e uma queda, em 2016 (23,21\%). Casos de trabalho infantil também foram notificados, chegando a corresponder a $1,31 \%$ das notificações de violência do ano de 2013 , o que representa 28 crianças. Foram notificados, ainda, casos de tortura, com 2,41\%, em 2009, e 4,41\%, em 2015.

A natureza da agressão mais ocorrente em todos os anos foi por meio do uso de força corporal/espancamento, chegando a 45,37\% das ocorrências, em 2016. Arma de fogo foi a natureza da agressão em $25,86 \%$ dos casos, no ano de
$2009 ; 17,67 \% ; 22,49 \% ; 18,58 \%$ e $21,92 \%$ das ocorrências notificadas em 2010, 2012, 2014, e 2016 respectivamente. O caso de violência sexual mais notificado em todos os anos foi o estupro, distribuindo-se em 4,56\% (2009); 9,38\% (2010); $12,19 \%$ (2011); 13,53\% (2012); 20,83\% (2013); 22,56\% (2014); 22,34\% (2015) e 17,65\% (2016) das notificações.

No que diz respeito à natureza da lesão, conforme a Tabela 3, observa-se um alto percentual de casos de corte/ perfuração/laceração, no ano de 2009, quando representou $50,44 \%$ dos casos notificados, podendo-se observar uma queda nos anos seguintes: $34,49 \%$, em $2010 ; 38,12 \%$, em 2012; chegando a $28,46 \%$, em 2014. Os anos de 2015 e 2016 não puderam ser analisados por conta da mudança ocorrida na ficha de notificação. Houve uma diminuição no percentual de politraumatismos, bem como traumatismos cranioencefálicos, $54,00 \%$ e $60,96 \%$, respectivamente. Quanto à parte do corpo atingida, a cabeça/face apresentou o maior percentual. Ainda que tenha diminuído durante os anos, foram responsáveis por $39,67 \%$ das ocorrências, em 2009 , e 19,59\%, em 2016. Observa-se grande número de casos em que múltiplos órgãos/regiões foram atingidos, chegando a corresponder a 13,66\% das ocorrências (2012). Mais uma vez, observa-se o alto percentual de ignorados, chegando a ter o maior percentual entre todas as categorias no ano de $2016(23,46 \%)$.

Conforme apresentado na Tabela 4 , no que diz respeito ao provável agressor, o sexo masculino predominou em todos os anos, inclusive com crescimento, passando de $37,27 \%$, em 2009, para 53,98\%, em 2016. As mulheres como prováveis agressoras também apresentaram aumento: $6,46 \%$ das ocorrências do ano de 2009 e 10,06\%, em 2016. Para esta análise, é importante relacionarmos o percentual ao número de casos notificados, uma vez que o número de notificações cresceu, na série estudada (exceto, em 2015).

Quanto ao vínculo ou grau de parentesco do provável agressor com a pessoa atendida, os pais foram os prováveis agressores em 12,17\% dos casos, em 2009, e 14,16\%, em 2016.

\section{DISCUSSÃO}

No quadro de violência contra a criança e o adolescente, na Bahia, pode-se observar um aumento das notificações de violências, no período (exceto no ano de 2015), enquanto sua caracterização contribui para o entendimento da violência e para o seu enfrentamento, além de servir para monitorar o sistema VIVA e subsidiar a formulação de políticas públicas. Porém, o alto número de informações ignoradas e/ou deixadas em branco é preocupante, uma vez que não permite uma análise aprofundada das ocorrências. Faz-se interessante analisar quais fatores podem estar influenciando o profissional notificador e como reverter este quadro.

Os achados quanto à raça/cor predominante nas vítimas são corroborados por outros estudos ${ }^{10,14}$ e pelo Instituto de Pesquisa Estatística Aplicada (IPEA) ${ }^{7}$, cujos dados apontam que, 
Tabela 3. Caracterização da violência contra crianças e adolescentes segundo tipologia, Bahia, 2009-2016

\begin{tabular}{|c|c|c|c|c|c|c|c|c|}
\hline & $\begin{array}{l}2009 \\
\mathrm{n}=789 \\
\%\end{array}$ & $\begin{array}{c}2010 \\
\mathrm{n}=1183 \\
\%\end{array}$ & $\begin{array}{c}2011 \\
\mathrm{n}=1378 \\
\%\end{array}$ & $\begin{array}{c}2012 \\
\mathrm{n}=1574 \\
\%\end{array}$ & $\begin{array}{c}2013 \\
\mathrm{n}=2136 \\
\%\end{array}$ & $\begin{array}{c}2014 \\
\mathrm{n}=2460 \\
\%\end{array}$ & $\begin{array}{c}2015 \\
\mathrm{n}=2131 \\
\%\end{array}$ & $\begin{array}{c}2016 \\
\mathrm{n}=2464 \\
\%\end{array}$ \\
\hline \multicolumn{9}{|l|}{ Tipo de violência* } \\
\hline Violência física & 86,82 & 67,71 & 72,28 & 73,82 & 66,29 & 69,76 & 71,61 & 76,38 \\
\hline Violência psicológica/moral & 12,93 & 21,81 & 18,14 & 24,71 & 21,54 & 17,8 & 23,09 & 18,55 \\
\hline Tortura & 2,41 & 2,2 & 2,25 & 1,91 & 1,5 & 2,64 & 4,41 & 3,33 \\
\hline Negligência/abandono & 4,69 & 17,16 & 13,57 & 6,61 & 7,35 & 4,72 & 6,1 & 6,53 \\
\hline Trabalho infantil & 0,51 & 0,93 & 0,29 & 0,7 & 1,31 & 0,69 & 0,42 & 0,89 \\
\hline Outro tipo de violência & 1,65 & 3,38 & 1,82 & 1,6 & 2,25 & 1,82 & 1,6 & 2,39 \\
\hline \multicolumn{9}{|l|}{ Natureza da agressão } \\
\hline Força corporal/espancamento & 43,35 & 35,16 & 36,5 & 34,37 & 33,38 & 40,98 & 42,14 & 45,37 \\
\hline Substância/Objeto quente & 0,63 & 1,61 & 1,89 & 1,65 & 2,01 & 1,1 & 0,84 & 1,54 \\
\hline Envenenamento & 0,13 & 0,85 & 0,15 & 0,57 & 0,33 & 0,08 & 0,33 & 1,18 \\
\hline Arma de fogo & 25,86 & 17,67 & 19,81 & 22,49 & 19,48 & 18,58 & 18,4 & 21,92 \\
\hline Ameaça & 7,73 & 12,51 & 8,35 & 12,96 & 12,36 & 13,29 & 15,72 & 13,19 \\
\hline Outro meio de agressão & 5,7 & 8,96 & 4,79 & 6,93 & 5,81 & 5,69 & 5,21 & 8,4 \\
\hline \multicolumn{9}{|l|}{ Violência sexual* } \\
\hline Assédio sexual & 2,79 & 5,41 & 4,72 & 5,84 & 6,41 & 4,92 & 7,51 & 5,88 \\
\hline Estupro & 4,56 & 9,38 & 12,19 & 13,53 & 20,83 & 22,56 & 22,34 & 17,65 \\
\hline Atentado violento ao pudor & 3,8 & 2,7 & 2,76 & 2,8 & 2,48 & 1,46 & 0 & 0 \\
\hline Pornografia infantil & 0,51 & 0,76 & 0,87 & 0,7 & 0,84 & 0,77 & 1,31 & 0,61 \\
\hline Traumatismo dentário & 0,25 & 0,08 & 0,07 & 0,19 & 0,23 & 0,41 & S.I & S.I \\
\hline Traumatismo cranioencefálico & 2,92 & 1,69 & 1,09 & 1,02 & 1,97 & 1,14 & S.I & S.I \\
\hline Politraumatismo & 9,13 & 2,7 & 1,89 & 3,3 & 3,46 & 3,17 & S.I & S.I \\
\hline Intoxicação & 0,38 & 0,85 & 0,51 & 0,95 & 0,66 & 0,24 & S.I & S.I \\
\hline Queimadura & 0,76 & 1,35 & 2,69 & 1,72 & 2,53 & 0,93 & S.I & S.I \\
\hline Outros & 8,24 & 8,53 & 5,08 & 8,83 & 6,93 & 5,08 & S.I & S.I \\
\hline Não se aplica & 7,35 & 14,45 & 14,51 & 15,37 & 24,39 & 17,52 & S.I & S.I \\
\hline Ignorado/* & 5,58 & 18,37 & 16,46 & 13,61 & 12,3 & 26,46 & S.I & S.I \\
\hline \multicolumn{9}{|l|}{ Parte do corpo atingida } \\
\hline Cabeça/face & 39,67 & 23,92 & 25,04 & 23,82 & 20,08 & 19,59 & S.I & S.I \\
\hline Pescoço & 2,79 & 1,69 & 2,03 & 1,59 & 1,73 & 1,26 & S.I & S.I \\
\hline Boca/dentes & 0,51 & 0,08 & 0,36 & 0,38 & 0,47 & 0,49 & S.I & S.I \\
\hline Coluna/medula & 0,89 & 0,76 & 0,44 & 0,64 & 0,61 & 0,41 & S.I & S.I \\
\hline Tórax/dorso & 4,56 & 3,72 & 4,57 & 5,15 & 4,96 & 5,45 & S.I & S.I \\
\hline Abdome & 2,28 & 2,45 & 2,32 & 2,35 & 2,29 & 2,52 & S.I & S.I \\
\hline Quadril/pelve & 1,27 & 1,1 & 0,87 & 0,7 & 1,08 & 0,73 & S.I & S.I \\
\hline Membros superiores & 10,52 & 6,51 & 7,33 & 8,07 & 8,19 & 7,6 & S.I & S.I \\
\hline Membros inferiores & 8,87 & 3,97 & 5,22 & 5,91 & 4,45 & 5,16 & S.I & S.I \\
\hline Órgãos genitais/ânus & 7,73 & 6,34 & 7,69 & 8,89 & 8,33 & 5,08 & S.I & S.I \\
\hline Múltiplos órgãos/regiões & 7,73 & 10,99 & 13,64 & 13,66 & 12,27 & 9,88 & S.I & S.I \\
\hline
\end{tabular}

Fonte: VIVA/SVS/MS 
$71,5 \%$ das pessoas assassinadas, por ano, no Brasil, são negras. Segundo o Instituto Brasileiro de Geografia e Estatística (IBGE) $^{12}$, de 2012 a 2016, houve um aumento no número de pessoas autodeclaradas pardas e pretas, enquanto o número de pessoas brancas diminuiu, mudança essa também perceptível nos achados deste artigo.

De 2009 a 2012, as vítimas do sexo masculino foram predominantes, porém este quadro se inverte a partir de 2013, quando a maioria das vítimas passa a ser do sexo feminino e mantém-se constante nos anos seguintes. A prevalência do sexo feminino entre as vítimas de violência infantil é observada no estudo de Santos ${ }^{14}$. Segundo o Mapa de Violência 2015, houve um aumento de $159 \%$ na taxa de homicídios de mulheres no Estado da Bahia, entre 2006 e 2013, ficando este atrás apenas dos estados de Roraima $(343,9 \%)$ e Paraíba $(229,2 \%)^{15}$. O Atlas da Violência $2018^{7}$ traz que, muitas mulheres vítimas de homicídio foram antes vítimas de outras violências, tornando estes agravos evitáveis. Ou seja, este aumento no número de meninas vítimas de violência infantil deve ser entendido como um alerta para o enfrentamento e quebra do ciclo da violência antes da sua possível evolução para o homicídio.

A zona de residência da vítima e a zona de ocorrência da violência majoritariamente urbanas são consistentes com os achados de outros estudos ${ }^{11,14}$, mas podem estar relacionadas com o fato de que o VIVA ainda não está consolidado uniformemente em todo o Brasil. O alto índice de cortes/perfurações como natureza da agressão também é corroborado por outros estudos, o que pode ser também relacionado com o alto índice de violência física ${ }^{9}$. A utilização de arma de fogo como meio de agressão apresenta percentuais preocupantes, sendo destacado também no Atlas da Violência $2018^{7}$, que relaciona a vitimização por arma de fogo com o crescimento da violência letal no país. Nesta análise, é também importante lembrar que jovens de 15 a 29 anos são as maiores vítimas de violência por arma de fogo ${ }^{16}$.

Os casos de estupro apresentaram aumento em todos os anos, porém, a partir de 2013, é mais expressivo: de 19,70\%, em 2012, para 27,43\%, em 2013. Alguns estudos trazem uma relação entre as vítimas do sexo feminino e a ocorrência da violência sexual ${ }^{4,9,10,13,14}$, sendo esta relação observada também em estudos acerca da violência em todas as faixas etárias ${ }^{5,11}$. De acordo com Andrade ${ }^{5}$, o processo de desenvolvimento no qual crianças e adolescentes se encontram, quando sua maturidade não está ainda completamente desenvolvida, dificulta o entendimento acerca dos mecanismos utilizados pelo agressor sexual, que envolve a imposição de autoridade no conflito de gênero e poder entre vítima e agressor. O perfil do agressor foi composto majoritariamente por homens, o que é corroborado por diversos estudos ${ }^{9,10,13}$.

Quanto à suspeita de uso de álcool por parte do agressor, observa-se um aumento ao longo dos anos. Bahia ${ }^{18}$ aponta o álcool como um fator de risco associado à violência, inclusive nas lesões autoprovocadas. O grande número de casos de violência ocorridos nas residências das vítimas é corroborado por outros estudos ${ }^{10} \mathrm{e}$, aliado ao grande percentual de pais classificados como prováveis agressores, transforma $\mathrm{o}$ local que deveria representar segurança e apoio para uma criança, em um local violento e adoecedor, principalmente para as vítimas de menor faixa etária, pois são ainda muito dependentes de seus pais e/ou responsáveis.

Tabela 4. Caracterização do provável agressor, Bahia, 2009-2016

\begin{tabular}{|c|c|c|c|c|c|c|c|c|}
\hline & $\begin{array}{c}2009 \\
\mathrm{n}=789 \\
\%\end{array}$ & $\begin{array}{c}2010 \\
\mathrm{n}=1183 \\
\%\end{array}$ & $\begin{array}{c}2011 \\
\mathrm{n}=1378 \\
\%\end{array}$ & $\begin{array}{c}2012 \\
\mathrm{n}=1574 \\
\%\end{array}$ & $\begin{array}{c}2013 \\
\mathrm{n}=2136 \\
\%\end{array}$ & $\begin{array}{c}2014 \\
\mathrm{n}=2460 \\
\%\end{array}$ & $\begin{array}{c}2015 \\
\mathrm{n}=2131 \\
\%\end{array}$ & $\begin{array}{c}2016 \\
\mathrm{n}=2464 \\
\%\end{array}$ \\
\hline Pai/Mãe & 12,17 & 33,64 & 23,66 & 20,96 & 19,01 & 16,3 & 16,8 & 14,16 \\
\hline Padrasto/Madrasta & 2,53 & 4,31 & 3,7 & 3,11 & 4,78 & 3,46 & 4,5 & 3 \\
\hline Cônjuge /Ex-Cônjuge/ Namorado(a)/Ex-Namorado(a) & 3,29 & 3,13 & 4,8 & 5,21 & 6,22 & 6,18 & 8,16 & 8,4 \\
\hline Amigos/conhecidos & 12,29 & 16,57 & 13,43 & 13,09 & 18,35 & 16,1 & 16,24 & 15,75 \\
\hline Desconhecido(a) & 13,31 & 10,06 & 9,58 & 9,4 & 14,19 & 11,59 & 13,14 & 17,98 \\
\hline Outros & 7,09 & 10,9 & 8,93 & 10,1 & 10,63 & 9,96 & 12,02 & 11,04 \\
\hline \multicolumn{9}{|l|}{ Sexo do provável autor da agressão } \\
\hline Masculino & 37,27 & 44,04 & 37,74 & 43,52 & 54,92 & 55,89 & 56,97 & 53,98 \\
\hline \multicolumn{9}{|l|}{ Suspeita de uso de álcool } \\
\hline Sim & 7,73 & 11,24 & 12,12 & 9,72 & 11,94 & 13,17 & 14,03 & 12,82 \\
\hline Não & 14,58 & 22,15 & 17,56 & 25,03 & 32,68 & 31,71 & 33,13 & 33,52 \\
\hline Ignorado & 77,69 & 66,61 & 70,32 & 65,25 & 55,39 & 55,12 & 52,84 & 53,66 \\
\hline
\end{tabular}

Fonte: VIVA/SVS/MS 
Neste estudo, encontramos um alto percentual de casos cujo encaminhamento realizado foi no ambiente hospitalar. Segundo o Ministério da Saúde ${ }^{6}$, as internações hospitalares devido às causas externas custam ao SUS cerca de 1,3 bilhão de reais por ano.

\section{CONCLUSÃo}

Os dados destacam o alto índice da violência infantil, principalmente contra as crianças do sexo feminino e de cor/ raça negra. $\mathrm{O}$ grande número de informações ignoradas é preocupante, pois dificulta uma análise mais fidedigna. Nesse sentido, entendemos que é preciso formação na temática e sensibilização por parte dos profissionais notificadores com vistas à compreensão de que seu papel é fundamental no enfrentamento à violência.

Os resultados encontrados por este estudo mostram a necessidade da realização de mais pesquisas acerca da violência, uma vez que o aprofundamento nesta temática pode diminuir a subnotificação e propor novas abordagens para o enfrentamento da violência. É importante ressaltar que, o fato de os dados não estarem disponíveis na plataforma de acesso público é uma limitação para a realização de pesquisas sobre o tema. Destacamos ainda a necessidade da participação da sociedade neste processo, com o objetivo de ampliar o debate acerca da temática e de promover um enfrentamento conjunto a toda forma de violência. Este enfrentamento passa pelo reconhecimento e combate das desigualdades presentes na sociedade, que, conforme aqui apontado e, também, na literatura, influenciam diretamente na ocorrência da violência.

\section{REFERÊNCIAS}

1. Minayo MCS. A inclusão da violência na agenda da saúde: trajetória histórica. Ciênc. saúde coletiva 2006; 11(Suppl): 1259-67.

2. Krug EG, Dahlberg LL, Mercy JA, Zwi AB, Lozano R. World report on violence and health. Geneva: World Health Organization; 2002

3. Stephan F, De Almeida AA, Salgado FS, Senra LX, Lourenço LM. Bullying e aspectos psicossociais: estudo bibliométrico. Temas psicol. 2013; 21(1):245-58.

4. Malta DC, Bernal RTI, Teixeira BDSM, Da Silva MMA, Freitas MIDF. Fatores associados a violências contra crianças em Serviços Sentinela de Urgência nas capitais brasileiras. Ciênc. saúde coletiva 2017; 22(9):2889-98.

5. Andrade, CSS, Costa MCO, Silva MLCA, Barreto CSLA. Notificação da violência física e sexual de crianças e adolescentes: o papel do sistema de vigilância de violências e acidentes/VIVA. Rev. Saúde Col. UEFS 2018; 8(1):46-53.

6. Ministério da Saúde (BR). Viva: Vigilância de Violências e Acidentes: 2013 e 2014. Brasília: MS; 2017.
7. Instituto de Pesquisa Estatística Aplicada (IPEA), Fórum Brasileiro de Segurança Pública (FBSP). Atlas da violência. Rio de Janeiro: IPEA/FBSP; 2018. [acesso em 11 mar 2021). Disponível em: https://www.ipea.gov.br/portal/index.php?option $=$ com_content\&view $=$ article $\&$ id $=33410 \&$ Itemid $=432$

8. Veloso MMX, Magalhães CMC, Dell'Aglio DD, Cabral IR, Gomes MM. Notificação da violência como estratégia de vigilância em saúde: perfil de uma metrópole do Brasil. Ciênc. Saúde Coletiva 2013; 18(5):1263-72.

9. Souza CS, Costa MCO, Assis SG, Musse JO, Sobrinho CN, Amaral MTR. Sistema de Vigilância de Violências e Acidentes/VIVA e a notificação da violência infanto-juvenil, no Sistema Único de Saúde/SUS de Feira de Santana-Bahia, Brasil. Ciênc. saúde coletiva 2014; 19(3):773-84.

10. Mascarenhas MDM, Malta DC, Silva MMA, Lima CM, Carvalho MGO, Oliveira VLA. Violência contra a criança: revelando o perfil dos atendimentos em serviços de emergência, Brasil, 2006 e 2007. Cad. Saúde Pública 2010; 26(2):347-57.

11. Souto RMCV, Barufaldi LA, Nico LS, De Freitas MG. Perfil epidemiológico do atendimento por violência nos serviços públicos de urgência e emergência em capitais brasileiras, Viva 2014. Ciênc. saúde coletiva 2017;22(9): 2811-23.

12. Instituto Brasileiro de Geografia e Estatística (IBGE.) População chega a 205,5 milhões, com menos brancos e mais pardos e pretos. [Internet]. Editoria: Estatísticas Sociais. 24 de novembro de 2017 (acesso em 11 mar 2021). Disponível em: https://agenciadenoticias.ibge.gov.br/agencia-noticias/2012agencia-de-noticias/noticias/18282-pnad-c-moradores

13. Farias MS, Souza CDS, Carneseca EC, Passos ADC, Vieira, EM. Caracterização das notificações de violência em crianças no município de Ribeirão Preto, São Paulo, no período 2006-2008. Epidemiol. serv. saúde 2016; 25(4):799-806.

14. Dos Santos TMB, Pitangui ACR, Bendo CB, Paiva SM, Cardoso MD, De Melo JPR, et al. Factors associated with the type of violence perpetrated against adolescents in the state of Pernambuco, Brazil. Child Abuse \& Neglect 2017; 67:216-17.

15. Waiselfisz JJ. Mapa da Violência 2015: homicídio de mulheres no Brasil. [Internet]. Brasília: FLACSO/CEBELA; 2015. (acesso em 11 mar 2021). Disponível em: https:// www.onumulheres.org.br/wp-content/uploads/2016/04/ MapaViolencia_2015_mulheres.pdf

16. Waiselfisz JJ. Mapa da Violência 2016: homicídios por armas de fogo no Brasil. [Internet]. Brasília: FLACSO/ CEBELA, 2016. (acesso em 11 mar 2021). Disponível em: https://flacso.org.br/files/2016/08/Mapa2016_armas_web-1.pdf

17. Malta DC, Bernal RTI, Pugedo FSF, Lima CM, Mascarenhas MDM, Jorge AO, et al. Violências contra adoles- 
centes nas capitais brasileiras, segundo inquérito em serviços de urgência. Ciênc. Saúde Coletiva 2017; 22(9):2899-2908.

18. Bahia CA, Avanci JQ, Pinto LW, Minayo MCDS. Lesão autoprovocada em todos os ciclos da vida: perfil das vítimas em serviços de urgência e emergência de capitais do Brasil. Ciênc. Saúde Coletiva 2017; 22(9):2841-50.

\section{Agradecimentos}

Esta pesquisa foi financiada pela Fundação de Amparo à Pesquisa do Estado da Bahia (FAPESB), através do Programa de Iniciação Científica da Universidade do Estado da Bahia (UNEB). Os dados foram disponibilizados pelo Ministério da Saúde, por meio da Secretaria de Vigilância em Saúde.

\section{ABSTRACT}

This study aimed to characterize, through a historical serie, the frame of violence in the state of Bahia by analyzing the notifications made by the public health services. The data was obtained from the Violence and Accidents Vigilance System (VIVA Contínuo) from 2009 to 2016 totalising 14,115 reported cases. The data showed a rise of $212.30 \%$ in the notifications done between 2009 to 2016. Regarding the the victims, the age group of 10 to 19 years old prevailed in all analysed years, the same goes for the presence of victims selfdeclared as black. The most frequently type was physical violence. The statistical analysis was made by the software Minitab18. The findings of this study are worrysome and shows the magnitude of the violence against children and adolescents as well as highlight their specificities and the importance of facing this health problem.

Keywords: Violence; Health services; Child; Adolescent.

\section{RESUMEN}

El estudio tuvo como objetivo caracterizar, a través de una serie histórica, el panorama de la violencia contra la niñez y adolescencia en el estado de Bahía a través del análisis de las notificaciones realizadas por los servicios de salud. Se utilizaron datos del Sistema de Vigilancia de Violencia y Accidentes (VIVA Contínuo) durante los años 2009 a 2016, totalizando 14.115 casos notificados. Los datos arrojaron un incremento del $212,30 \%$ en las notificaciones, entre 2009 y 2016. En cuanto a las víctimas, predominó el grupo de edad de 10 a 19 años en todos los años analizados, así como la presencia de víctimas autodeclaradas negras. El tipo más frecuente fue la violencia física. Para el análisis estadístico se utilizó el software Minitab18. Los hallazgos de este estudio son preocupantes y demuestran la magnitud de la violencia contra la niñez y la adolescencia, además de resaltar sus especificidades y la importancia de combatir este problema de salud.

Palabras Clave: Violencia; Niño; Servicios de salud; Adolescente. 